\title{
ROS-dependent HMGB1 secretion upregulates IL-8 in upper airway epithelial cells under hypoxic condition
}

\author{
HJ Min ${ }^{1}, \mathrm{~J}-\mathrm{H} \mathrm{Kim}{ }^{2}, \mathrm{JE} \mathrm{Yoo}{ }^{2,3}, \mathrm{~J}-\mathrm{H} \mathrm{Oh}^{4}, \mathrm{KS} \mathrm{Kim}{ }^{1}, \mathrm{~J}-\mathrm{H} \mathrm{Yoon}^{2,3,5}$ and C-H Kim ${ }^{3,5}$
}

High-mobility group box 1 (HMGB1) mediates various functions according to the location. We tried to investigate the role of HMGB1 in upper airway under hypoxic conditions. We cultured primary normal human nasal epithelium (NHNE) cells under hypoxic conditions and evaluated the movement of HMGB1 by western blotting, immunofluorescence, and enzyme-linked immunosorbent assay (ELISA). Reactive oxygen species (ROS) level was evaluated to estimate the translocation mechanism of HMGB1. The role of secreted HMGB1 was evaluated by ELISA assay. Furthermore, we collected human nasal mucosa samples and nasal lavage fluids from patients conditioned under hypoxic and nonhypoxic environment, and compared the expression of HMGB1 in human nasal mucosa samples by immunohistochemistry and the levels of HMGB1 in lavage fluids using ELISA assay. Hypoxia induced translocation of HMGB1 into the extracellular area and it was dependent on ROS produced by dual oxidase 2. Secreted HMGB1 was involved in the upregulation of interleukin (IL)-8. In human samples, HMGB1 was translocated from nucleus to the cytoplasm in hypoxic-conditioned nasal mucosa. HMGB1 was increased in nasal lavage samples of chronic rhinosinusitis patients, whose sinus mucosa was supposed to be hypoxic as compared with controls. We suggest that HMGB1 is secreted in hypoxic condition via ROS-dependent mechanism and secreted HMGB1 participates in IL-8 upregulation mediating inflammatory response.

\section{INTRODUCTION}

Nuclear high-mobility group box 1 (HMGB1) functions as a DNA chaperone. ${ }^{1}$ HMGB1 can be released into the extracellular space via an active process following pro-inflammatory stimulation and passively into the extracellular space following necrosis via the leaky cell membrane. ${ }^{1,2}$ In the extracellular space, HMGB1 binds to transmembrane receptors, including Toll-like receptor (TLR) 2 and TLR4, and the receptor for advanced glycation end products. ${ }^{3,4}$ Binding of HMGB1 to receptors triggers activation of proinflammatory signalling pathways and induces pleiotropic effects depending on the cell type $^{5-7}$ Recently, not only extracellular HMGB1 but also cytoplasmic HMGB1 has been found to function as an autophagy regulator and a chaperone-like molecule. ${ }^{8,9}$ In addition, the translocation of HMGB1 from the nucleus to the extracellular area was dependent on the posttranslational modifications such as phophorylation, acetylation, and oxidation. ${ }^{1,2,9}$ In our previous study, we proved that reactive oxygen species (ROS) was important in translocation of HMGB1. ${ }^{10}$

Hypoxia is defined as a low level of oxygen in the environment and oxygen concentrations of $<5 \%$ are usually considered to be hypoxic. ${ }^{11,12}$ In the nasal cavity, many pathologic diseases are related to tissue hypoxia. Chronic sinusitis is characterized by anatomical obstruction of the sinus natural ostium with chronic/recurrent infectious conditions, whereas the development of nasal polyps is associated with hypoxic conditions as well. ${ }^{11}$ Acute sinusitis was associated with a lower oxygen concentration and the severity of sinusitis based on computed tomography scans was inversely correlated with the oxygen concentrations. ${ }^{13}$ Furthermore, hypoxia is

${ }^{1}$ Department of Otorhinolaryngology-Head and Neck Surgery, Chung-Ang University College of Medicine, Seoul, Republic of Korea. ${ }^{2}$ The Research Center for Human Natural Defense System, Yonsei University College of Medicine, Seoul, Republic of Korea. ${ }^{3}$ The Airway Mucus Institute, Yonsei University College of Medicine, Seoul, Republic of Korea. ${ }^{4}$ Brain Korea 21 PLUS Project for Medical Science, Yonsei University College of Medicine, Seoul, Republic of Korea and ${ }^{5}$ Department of Otorhinolaryngology, Yonsei University College of Medicine, Seoul, Republic of Korea. Correspondence: C-H Kim (ENTMAN@yuhs.ac) 
associated with diminished mucocilliary clearance, which ultimately impairs the nasal epithelial defense mechanism. ${ }^{14}$

In the nasal cavity, HMGB1 was detected in nasal lavage fluids and the HMGB1 levels were correlated with the Lund Mackay score in chronic rhinosinusitis patients. ${ }^{15}$ As HMGB1 translocates from the nucleus to the cytoplasm in a hypoxic tissue environment and is eventually released into the extracellular space, HMGB1 may serve as a molecular marker for detecting hypoxia-associated tissue damage in organs. ${ }^{16,17}$ These previous findings suggest that HMGB1 may have a role in upper airway inflammatory diseases that originate in a hypoxic tissue environment; however, no studies have investigated the role of HMGB1 in the upper airway under hypoxic conditions. Thus, the current study was undertaken to investigate the effects of hypoxia on the expression and translocation of HMGB1 protein in the upper airway epithelium and to determine the underlying mechanism behind this expression and translocation following exposure to hypoxic conditions in the upper airway epithelium. Furthermore, we sought to unveil the role of HMGB1 protein in hypoxia-associated upper airway inflammatory diseases.

\section{RESULTS}

\section{Hypoxia induces translocation of HMGB1 in primary upper} airway epithelial cells

We found that hypoxia induced translocation of HMGB1 from the nucleus to the extracellular area in RPMI 2650 cells (Supplementary Figure S1 online). Next, we evaluated the effect of hypoxic stimulation on the expression level of HMBG1 in human primary nasal epithelial cells. Hypoxia mildly increased the amount of HMGB1 at both genetic and protein levels; however, it was not statistically significant (Figure 1a-c). To evaluate the location of HMGB1 protein, we performed immunofluorescence assay and found that cytoplasmic HMGB1 was increased after hypoxic stimulation (Figure 1d). Furthermore, we collected the supernatants from normal human nasal epithelium (NHNE) cells incubated in a hypoxic environment, to investigate HMGB1 secretion into the extracellular space. Western blotting analysis revealed that secretion of extracellular HMGB1 was increased after $8 \mathrm{~h}$ of hypoxic stimulation (Figure 1e) and quantification of the protein levels from three independent repeated western blotting indicated that this increase was statistically significant (Figure 1f).

\section{HMGB1 is secreted into extracellular area through hypoxia- induced ROS}

We tried to figure out whether ROS was involved in hypoxiainduced HMGB1 secretion mechanism and performed dichlorofluorescin diacetate staining. The ROS levels were increased after hypoxic stimulation and this increase in ROS was blocked with pretreatment of the ROS scavenger $\mathrm{N}$-acetyl cysteine (NAC) (Figure 2a). Mean fluorescence intensities were calculated from repetition of the experiments. Hypoxia significantly increased the ROS levels compared with the ambient control conditions and pretreatment of NAC inhibited hypoxia-induced upregulation of ROS levels (Figure 2b). When we evaluated the viability of NHNE cells after hypoxia with/without NAC pretreatment, it did not affect cell viability (Supplementary Figure S2). Next, we performed immunofluorescence assay and found that cytoplasmic translocation of HMGB1 was reduced with the pretreatment of NAC, suggesting that cytoplasmic translocation of HMGB1 is directly associated with the increase of ROS (Figure 2c). To determine whether extracellular HMGB1 secretion from NHNE cells was associated with the increase of ROS, we collected apical culture supernatants with/without NAC pretreatment and performed western blotting assay. The amount of extracellular HMGB1 protein was decreased in cultures pretreated with NAC compared with cultures without NAC (Figure 2d). By enzyme-linked immunosorbent assay (ELISA), we found the same result that HMGB1 was secreted into the extracellular area after hypoxic stimulation and it was abolished with pretreatment of NAC (Figure 2e), suggesting that ROS generated under hypoxic conditions is responsible for the secretion of HMGB1 in NHNE cells.

\section{DUOX2 has a significant role in hypoxia-induced HMGB1 secretion}

ROS can be generated by a range of mitochondrial chain transporter and NADPH oxidase enzymes. Dual oxidase (DUOX) 1 and 2, subtypes of NADPH oxidase enzymes, are expressed in epithelial cells of various tissues including human nasal epithelial cells and, previously, we have found that DUOXs had an important role in generation of ROS under upper airway inflammatory condition. ${ }^{18}$ Therefore, we then evaluated whether DUOX1 or DUOX2 is important in the observed hypoxia-induced ROS generation in nasal epithelial cells. We used short hairpin RNA for DUOX1 and DUOX2, to knock down each gene in NHNE cells. In our short hairpin RNA system, genetic expression of DUOX1 and DUOX2 was reduced $\sim 50 \%$ and $70 \%$, respectively (Figure 3a). ROS production was significantly reduced in DUOX-depleted NHNE cells, but the decrease was only significant in the DUOX2-depleted NHNE cells (Figure $\mathbf{3 b}, \mathbf{c}$ ). The secretion of HMGB1 following hypoxia treatment was also significantly reduced only in shDUOX2-transfected NHNE cells by ELISA assay (Figure 3d).

\section{HMGB1 increases IL-8 secretion in NHNE cells after hypoxic stimulation}

To elucidate the role of NHNE cell-secreted HMGB1, we collected the apical culture supernatants. Interleukin (IL)-8 was increased in hypoxia-conditioned apical media and this increase was abolished by pretreatment of cells with NAC, suggesting that the effects on IL-8 were dependent on ROS generation (Figure 4a).

Next, we incubated NHNE cells with $4 \mu \mathrm{g} \mathrm{ml}^{-1}$ mammalian recombinant HMGB1 (rHMGB1).

The concentration of IL-8 in the apical culture supernatants was significantly increased with the rHMGB1 treatment 
a

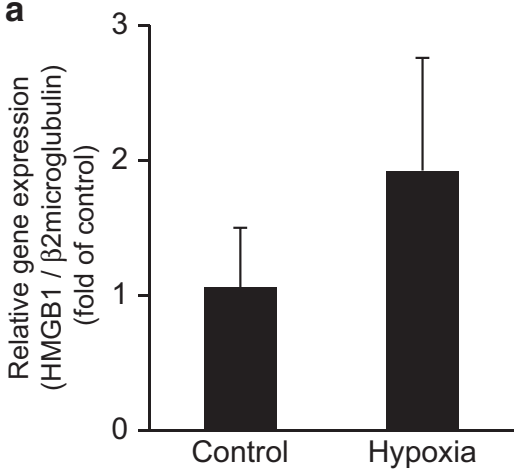

b

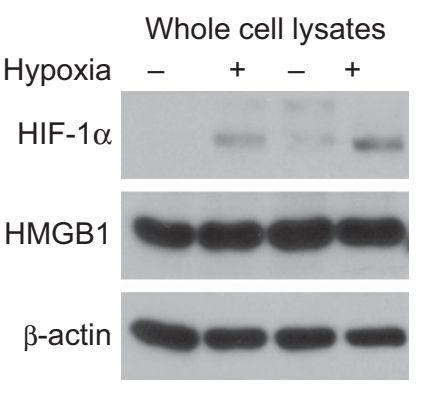

e
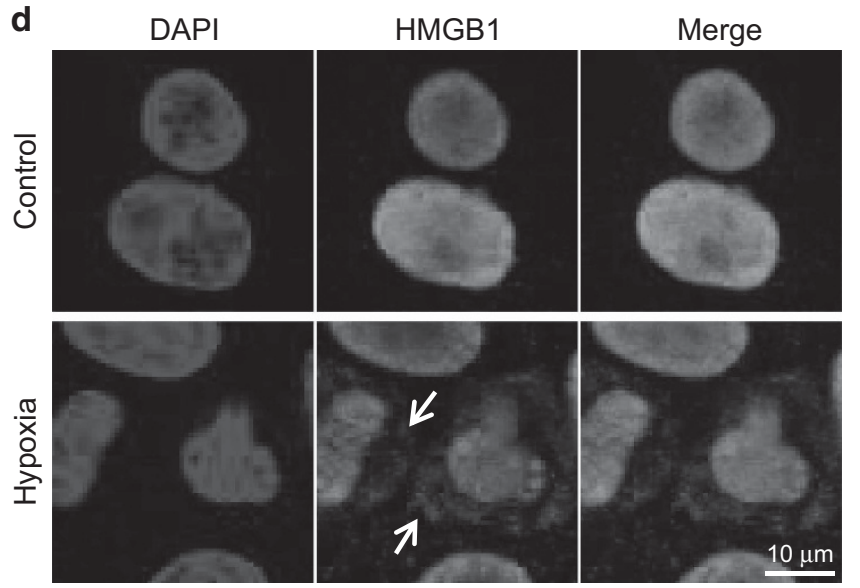

Figure 1 Hypoxia induces secretion of high-mobility group box 1 (HMGB1) protein in normal human nasal epithelium (NHNE) cells. (a) NHNE cells were incubated under hypoxic conditions for $8 \mathrm{~h}$ and real-time PCR was performed to evaluate relative amount of HMGB1 mRNA. (b) NHNE cells were incubated under hypoxic conditions and western blot assay was performed to evaluate the relative amount of HMGB1 protein. (c) Relative band intensities of HMGB1 protein in whole-cell lysates were calculated and compared between control and hypoxic conditions $(N=3)$. (d) NHNE cells were incubated under hypoxic conditions and then immunofluorescence assay was performed for HMGB1 (red) and with 4',6-diamidino-2-phenylindole (DAPI, blue) for DNA. Arrows indicate cytoplasmic HMGB1 protein. (e) Apical culture supernatants of NHNE cells were collected and concentrated. A western blot assay was performed to compare the HMGB1 protein level secreted into the extracellular space. (f) Relative band intensities of HMGB1 protein in culture supernatants were calculated and compared between control and hypoxic conditions $(N=3)$. ${ }^{\star} P<0.05$.

(Figure 4b). Flagellin is known to be a stimulator of IL-8 and used as a positive control. ${ }^{18}$ To determine whether the induction of IL-8 originated from extracellular HMGB1 secreted from the surrounding NHNE cells, we performed a pull-down assay for secreted HMGB1. NHNE cells were incubated under hypoxic conditions for $8 \mathrm{~h}$ and apical culture supernatants were collected. In the collected supernatants, HMGB1 was pulled down with anti-HMGB1 antibody. Western blot assays of the precipitated samples revealed that hypoxia-induced extracellular HMGB1 was effectively pulled down with the anti-HMGB1 antibody (Figure 4c). We then treated NHNE cells with the remnant culture supernatants for $24 \mathrm{~h}$ and the amount of IL-8 was measured by ELISA assay. Control culture supernatants, which were not depleted of HMGB1 by the anti-HMGB1 antibody, increased IL-8 production as expected; however, the HMGB1-depleted culture supernatant failed to increase IL- 8 production in NHNE cells (Figure 4d), indicating that secreted HMGB1 protein has a major role in the induction of IL-8. To confirm this result, we incubated NHNE cells under hypoxic conditions in the presence or absence of the anti-HMGB1 blocking antibody, which binds to extracellular HMGB1 and inhibits the function of secreted HMGB1 protein. Similar to our previous results, hypoxic conditions significantly increased the amount of IL-8 production and pretreatment with anti-HMGB1 blocking antibody abrogated this effect (Figure 4e).

\section{HMGB1 is secreted into extracellular space in hypoxic human sino-nasal mucosa}

To explore the implications of our findings in vivo, we collected human sino-nasal mucosa from patients who underwent endoscopic sinus surgery (ESS) (Table 1). Control nasal mucosa and hypoxic nasal mucosa were collected and immunohistochemistry was performed on these samples. HMGB1 was expressed in both epithelial and subepithelial cells. Most of HMGB1 protein was observed in the nucleus of cells in control mucosa, but in hypoxic mucosa HMGB1 was translocated to the cytoplasm as revealed by positive immunostaining in the extranuclear area (Figure 5a). We compared the numbers of cytoplasmic HMGB1-stained epithelial cells using these human sino-nasal mucosa samples and found that the translocation rate was higher in 


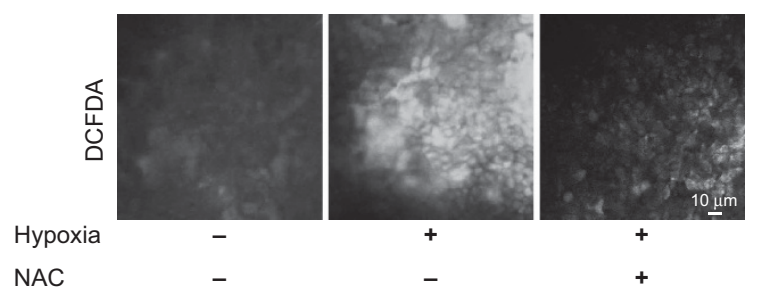

c

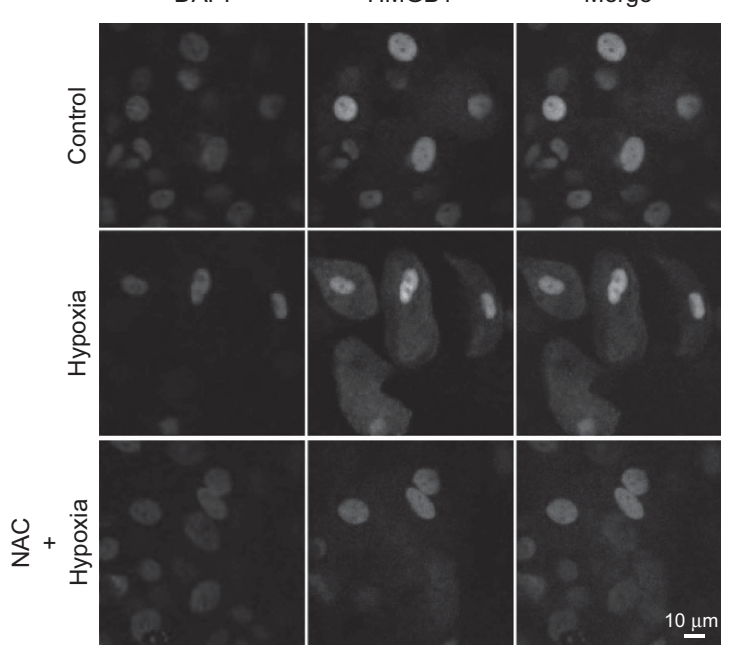

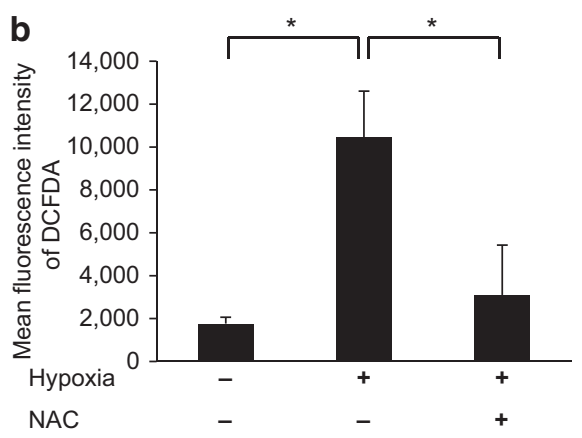

d

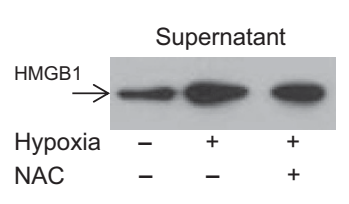

e

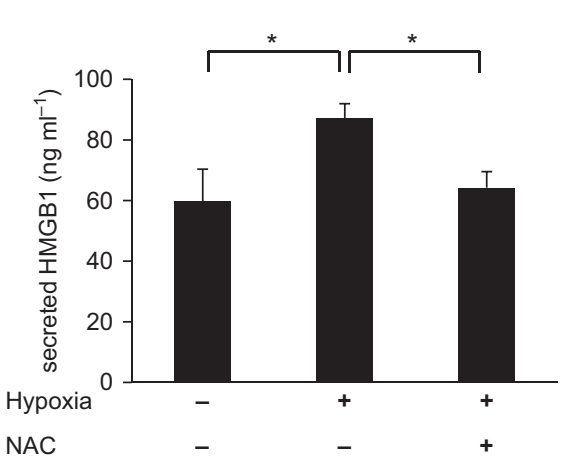

Figure 2 Hypoxia induces translocation of high-mobility group box 1 (HMGB1) via reaction oxygen species (ROS) production in normal human nasal epithelium (NHNE) cells. (a) NHNE cells that were incubated under hypoxic conditions in the presence or absence of $\mathrm{N}$-acetyl cysteine (NAC) treatment were stained with dichlorofluorescin diacetate (DCFDA) for $20 \mathrm{~min}$ at $37^{\circ} \mathrm{C}$ and stained cells were visualized by confocal microscopy. (b) Mean fluorescence was quantified using Image $J$ software. Results are shown as mean \pm s.e.m. $(N=3)$. ${ }^{*} P<0.05$. (c) NHNE cells were incubated under hypoxic conditions in the presence or absence of NAC pretreatment and an immunofluorescence assay was performed for HMGB1 (green) and 4',6diamidino-2-phenylindole (DAPI) staining (blue) for DNA. (d) Culture supernatants from NHNE cells were collected and concentrated. A western blot assay was performed, to compare the levels of HMGB1 protein secreted into the extracellular space after hypoxia with/without NAC pretreatment. (e) Enzyme-linked immnunosorbent assay (ELISA assay) using apical NHNE culture supernatant was performed to compare the levels of HMGB1 protein secreted into the extracellular space after hypoxia with/without NAC pretreatment. $(N=3) .{ }^{*} P<0.05$.

hypoxic human sino-nasal mucosa (Figure $5 \mathbf{b}$ ). In addition, we collected nasal lavage fluid from patients who underwent ESS. A total of 18 samples were obtained from hypoxic maxillary sinus and 17 samples were obtained from control maxillary sinus. ELISA assay using nasal lavage fluid revealed that HMGB1 was present in all nasal lavage fluids and the levels were significantly higher in patients with a totally obstructed sinus (hypoxic) compared with levels in the patients whose sinus was not obstructed (normoxic; Figure 5c). IL-8 was also detected in all nasal lavage fluid samples and the level of IL-8 was significantly higher in patients whose sinus was totally obstructed compared with the patients whose sinus was not obstructed (Figure 5d)

\section{DISCUSSION}

Hypoxia is closely related with inflammatory condition. In patients undergoing kidney transplantation, the renal expression of TLR4 was correlated with ischemic injury influencing on higher rate of immediate graft function. ${ }^{19}$ In the lung, pulmonary cytokine levels and TLR expression was correlated with the ischemic injury. ${ }^{20}$ The shortage of oxygen supply upregulated inflammatory adipokines in fat, resulting insulin resistance. ${ }^{21}$ Many clinical studies have proved that hypoxia promotes inflammation and leads to the occurrence of various diseases.

HMGB1 is a nuclear protein and one of the unique structural characteristics of HMGB1 is the presence of three cysteine residues (Cys23, Cys45 [Box A], and Cys106 [Box B]), which are sensitive to redox potential. ${ }^{22}$ On secretion to the extracellular space, the redox state of these three cysteine residues determines the activity of the HMGB1 protein. ${ }^{23,24}$ Upper airway inflammation is also related with hypoxic condition and it has been found that hypoxia upregulates inflammatory molecules in nasal epithelium and increases permeability and tissue remodeling. ${ }^{25-27}$ In the current study, we speculated that hypoxic exposure of NHNE cells would influence the redox status of the environment, affecting the location and function of HMGB1. Under our experimental 
a

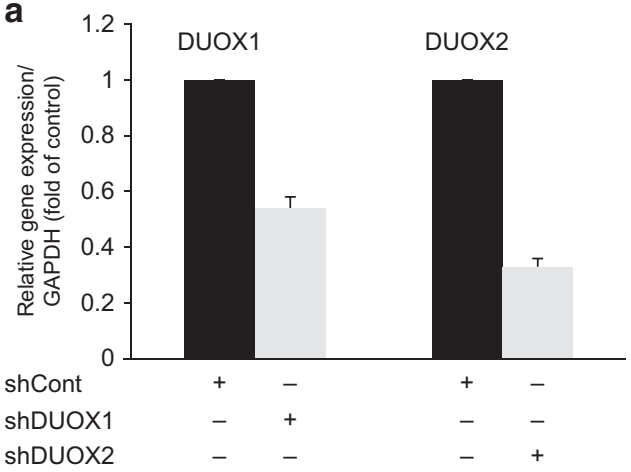

C

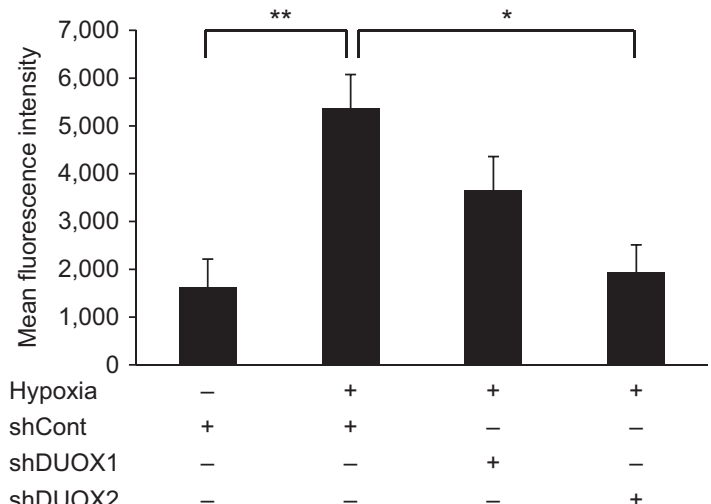

b

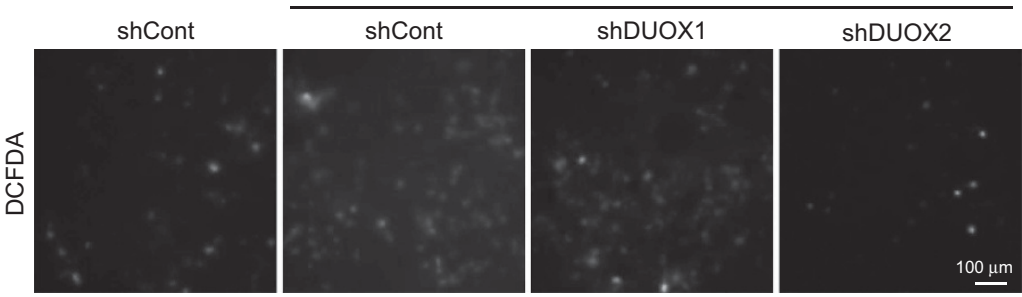

d

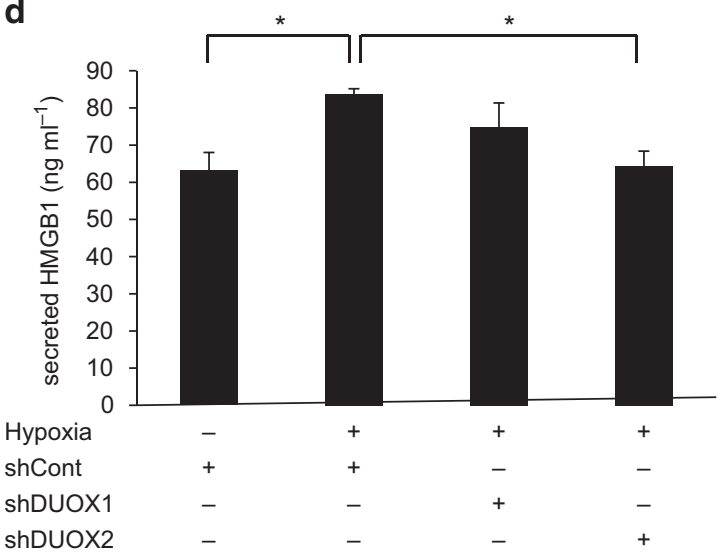

Figure 3 Dual oxidase 2 (DUOX2) has a role in hypoxia-induced secretion of high-mobility group box 1 (HMGB1). (a) Gene expression of dual oxidase 1 (DUOX1) and DUOX2 relative to glyceraldehyde 3-phosphate dehydrogenase (GAPDH) expression in normal human nasal epithelium (NHNE) cells after transfection with shCont, shDUOX1, and shDUOX2. (b) NHNE cells transfected with shCont, shDUOX1, or shDUOX2 were incubated under hypoxic conditions for $8 \mathrm{~h}$ and stained with dichlorofluorescin diacetate (DCFDA) for $20 \mathrm{~min}$ at $37^{\circ} \mathrm{C}$, to visualize reactive oxygen species (ROS). (c) Mean fluorescence of the images in (b) was quantified using Image J software. Results are presented as mean \pm s.e.m. ( $N=3$ ). (d) HMGB1 enzyme-linked immnunosorbent assay (ELISA) was performed to compare the HMGB1 protein levels secreted into extracellular space in the presence of short hairpin RNAs (shRNAs; $N=3$ ). ${ }^{*} P<0.05$ and ${ }^{* *} P<0.01$.

conditions ( $8 \mathrm{~h}$ of $1 \%$ oxygen), hypoxia increased the ROS levels, which, in turn, were associated with the translocation of HMGB1 protein to extracellular area through cytoplasm, and this is the first study, as far as we know, that revealed the relationship of hypoxia-induced ROS with the secretion of HMGB1 in the upper airway.

To date, the relationship between hypoxia and ROS levels remains contentious. Exposure to hypoxia increases the local environmental redox potential due to alteration of the production of short-lived ROS. ${ }^{28}$ Some studies proposed that ROS production depends on the concentration of oxygen, showing that mild hypoxia induces the production of ROS but severe hypoxia does not. ${ }^{29}$ Based on these findings, we expect that longer durations of hypoxia exposure or another concentration of oxygen in the environment may cause different results. Therefore, the redox status of secreted HMGB1 under various hypoxic conditions will be another interesting line of study. The possibility that different oxygen concentrations will change the redox potential of the HMGB1 structure discriminatively is reasonable and such changes may lead to a different function of HMGB1.
Using short hairpin RNA system, we have found that DUOX2 was the main source of ROS-mediated HMGB1 secretion under hypoxic condition. It was previously reported that DUOX2 generated ROS-mediated TLR2 and TLR4 activation in the upper airway epithelium. ${ }^{18}$ We have shown that DUOX2 but not DUOX1 was important in hypoxiainduced HMGB1 secretion. Furthermore, the fact that TLR2 and TLR4 are HMGB1 receptors further support our hypothesis that DUOX2 and HMGB1 might be important inflammatory mediators under hypoxic condition in the upper airway.

Surprisingly, in our experiments, treatment with mammalian rHMGB1 protein alone did not increase the production of other cytokines, such as tumor necrosis factor- $\alpha$ or IL-1 $\beta$, which are known to be general pro-inflammatory mediators in NHNE cells (data not shown). Instead, we found that treatment with mammalian-originated rHMGB1 protein induced the secretion of IL-8 (CXCL8), which functions as a chemoattractant for neutrophils. ${ }^{30}$ Hypoxia has been reported to increase the secretion of IL-8 in human nasal polyp fibroblasts; ${ }^{31}$ however, the mechanism has not yet been identified. Here we provide evidence that HMGB1 induces secretion of IL-8 in nasal epithelium under hypoxic condition. 
a

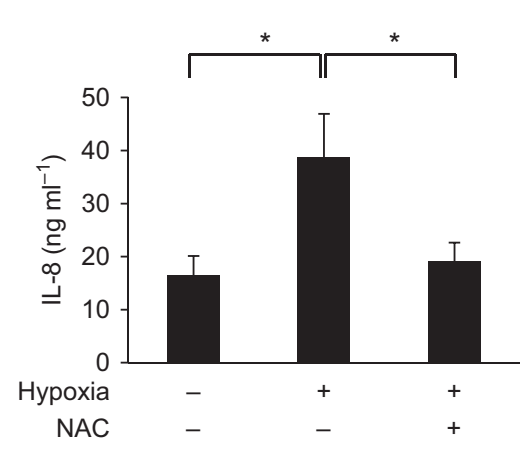

b
C

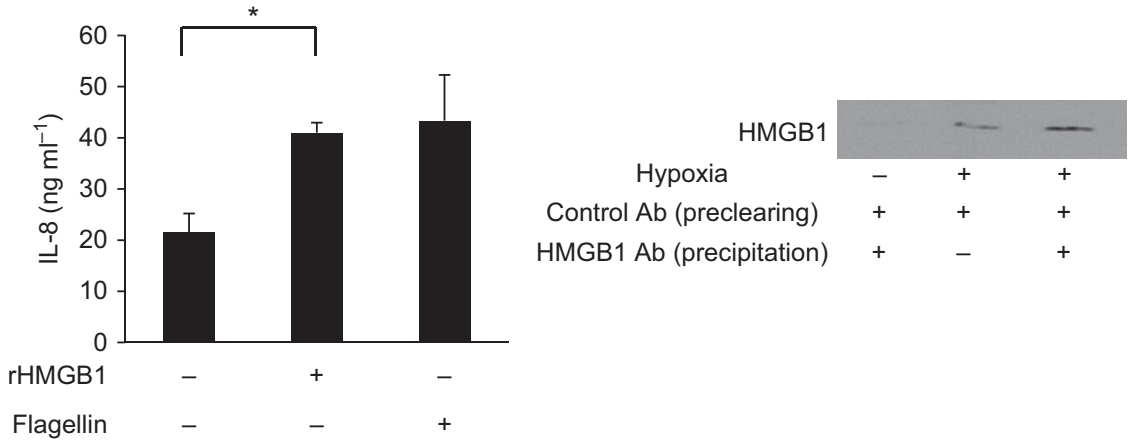

e

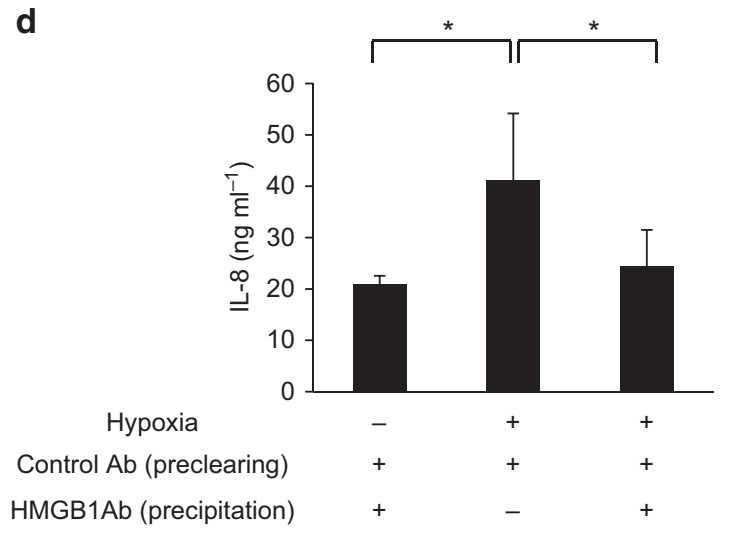

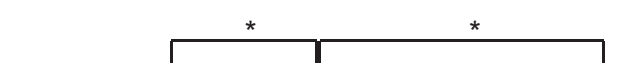

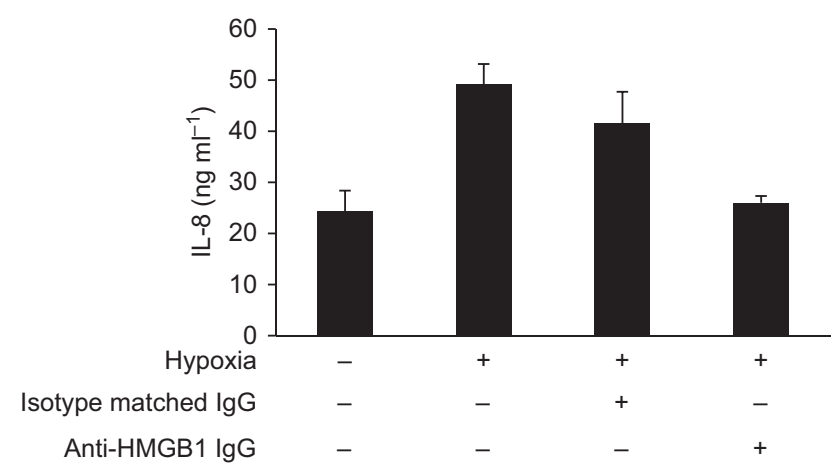

Figure 4 High-mobility group box 1 (HMGB1) increases interleukin (IL)-8 secretion in normal human nasal epithelium (NHNE) cells grown under hypoxic conditions. (a) NHNE cells were incubated under hypoxic condition with/without $\mathrm{N}$-acetyl cysteine (NAC) pretreatment. The amount of interleukin (IL)-8 secreted into apical culture medium was quantified by enzyme-linked immnunosorbent assay (ELISA). (b) NHNE cells were incubated with mammalian recombinant HMGB1 (rHMGB1) protein $\left(4 \mu \mathrm{g} \mathrm{ml}^{-1}\right)$ for $24 \mathrm{~h}$. Amounts of IL-8 in the apical supernatants produced by NHNE cells were determined by ELISA assay. Flagellin $\left(100 \mathrm{ng} \mathrm{ml}^{-1}\right)$ was used as a positive inducer of IL-8. (c) NHNE cells were incubated under hypoxic conditions for $8 \mathrm{~h}$ and then the apical culture medium was collected. HMGB1 protein that had been secreted into the apical culture medium was precipitated and depleted using anti-HMGB1 antibody. HMGB1-precipitated culture supernatants were separated and a western blot assay was performed to confirm the precipitation of secreted HMGB1 protein. (d) NHNE cells were incubated under hypoxic conditions for $8 \mathrm{~h}$ and apical culture medium, which might contain secreted HMGB1 protein, was collected. This culture supernatant was precleared using protein G-Sepharose or serially precleared and precipitated using anti-HMGB1 antibody, to remove secreted HMGB1 protein. Supernatants that were only precleared or that had had HMGB1 removed were applied into NHNE cells for $24 \mathrm{~h}$. Next, IL-8 produced in the apical supernatants by NHNE cells was determined by ELISA assay. (e) NHNE cells were incubated under hypoxic conditions with or without anti-HMGB1 blocking antibody for $24 \mathrm{~h}$. Apical culture medium was collected and IL-8 produced by NHNE cells was determined using ELISA assay. For all panels, results are shown as mean \pm s.e.m. $(N=3)$. ${ }^{\star} P<0.05$.

HMGB1 exerts proinflammatory activity only after forming complexes with lipopolysaccharide and pure recombinant HMGB1 only acts as a chemoattractant and a mitogen, ${ }^{32}$ in agreement with our findings. A previous study reported that anoxia induced various inflammatory cytokines in respiratory epithelial cells. ${ }^{33}$ Thus, we speculate that not pure HMGB1 protein itself, but HMGB1 protein in complex with other molecules in the culture supernatant, may increase the production of other cytokines (such as tumor necrosis factor- $\alpha$, which was not increased with pure recombinant HMGB1 protein stimulation) and propagate inflammation.

The novelty of our study is that we demonstrated original finding using upper airway epithelium cell line (Supplementary Figure S1) and primary nasal epithelial cells. In addition, our in vitro finding was verified using human nasal samples. Although we have tried to establish mouse model of nasal local hypoxic condition, we could not get appropriate models to confirm our in vitro experimental findings. Instead, as clinicians, we collected human nasal samples and evaluated the histologic finding, and the secretion of HMGB1 and IL-8 using nasal lavage samples. Corresponding to our in vitro data, HMGB1 was much more frequently observed in the cytoplasm of nasal epithelium exposed to hypoxia compared with normoxia and it was further increased in nasal lavage fluid samples along with the increase of IL-8. In addition, the absolute amount of HMGB1 in nasal lavage fluid was much higher than IL-8, which is a well-evaluated chemokine in upper airway inflammation, suggesting that HMGB1 might be another important biomarker in upper airway inflammation.

In conclusion, HMGB1 is secreted from nasal epithelium under hypoxic conditions both in vitro and in vivo. Furthermore, extracellular HMGB1 induced upregulation of IL-8 via an autocrine or paracrine pathway. These findings suggest that HMGB1 has a crucial role in the pathogenesis of hypoxiamediated upper airway inflammatory diseases. 
Table 1 Clinical characteristics of enrolled patients

\begin{tabular}{lcc}
\hline Parameter & $\begin{array}{c}\text { Value (hypoxic } \\
\text { condition) }\end{array}$ & $\begin{array}{c}\text { Value (non-hypoxic } \\
\text { condition) }\end{array}$ \\
\hline$N$ & 18 & 17 \\
Sex, male/female & $13 / 5$ & $11 / 6$ \\
Age, years & $49.11 \pm 16.83$ & $44.41 \pm 19.24$ \\
Body mass index, & $22.82 \pm 3.39$ & $26.19 \pm 3.36$ \\
kg m $^{-2}$ & & \\
Hospitalization & $2.52 \pm 0.51$ & $2.52 \pm 0.51$ \\
duration, days & $10(55.55)$ & $8(47.05)$ \\
$\begin{array}{l}\text { Presence of nasal } \\
\text { polyp, \% }\end{array}$ & $5.29 \pm 1.53$ & $5.05 \pm 2.01$ \\
Lund Mackay score & & \\
Concurrent medical \\
diseases
\end{tabular}

\section{METHODS}

Cell culture. NHNE cells were cultured as described previously. ${ }^{34}$ Passage- 2 NHNE cells $\left(1 \times 10^{5}\right.$ cells $)$ were seeded on a $24.5 \mathrm{~mm}$ Transwell clear-culture insert with a $0.45 \mathrm{~mm}$ pore size (Costar, Cambridge, MA). The culture medium was changed on day 1 and every other day until the cultures were submerged. The air-liquid interface was created by removing the apical medium. Subsequently, culture medium was changed daily and experiments were performed using NHNE cells grown with air-liquid interface for 14 days. RPMI 2650 cells were maintained in Eagle's minimum essential medium (Gibco, Karlsruhe, Germany) supplemented with $0.11 \mathrm{gl}^{-1}$ sodium pyruvate, $4 \mathrm{~mm}$ GlutaMAX, and $10 \%$ fetal bovine serum at $37^{\circ} \mathrm{C}$ in a $>95 \%$ humidified atmosphere of $5 \% \mathrm{CO}_{2}$ in air with media changes on alternate days following previous protocol. ${ }^{35}$ RPMI 2650 cells were used for experiment 2 days after cell splitting.

Hypoxia was induced by placing cells in a hypoxic incubator that maintains $1 \%$ oxygen for the indicated duration.

Nucleus and cytoplasm fractionation. Nucleus and cytoplasmic proteins were extracted using the Nuclear/Cytosol Fractionation Kit (BioVision, Milpitas, CA) according to the manufacturer's protocol. Briefly, cells were collected and lysed with CEB-A Mix (containing dithiothreitol and protease inhibitors) and CEB-B sequentially. After separation of the cytoplasmic proteins, NEB Mix (containing dithiothreitol and protease inhibitors) was added to extract the nuclear proteins.

Detection of secreted HMGB1 in cell culture supernatant. Apical culture supernatants from identical numbers of cells were collected and concentrated, to compare the amount of secreted HMGB1 proteins in the extracellular space. Cells $\left(5 \times 10^{5}\right)$ were seeded in a sixwell plate and maintained under hypoxic conditions for the indicated times. All of the cell culture supernatant (total $2 \mathrm{ml}$ ) was collected and concentrated using a Centrifugal Filter Unit (Millipore, Billerica, MA).

Western blot analysis. Forty micrograms of the protein samples was separated on a $12 \%$ SDS-polyacrylamide gel electrophoresis and then proteins were transferred to a nitrocellulose membrane. Primary antibodies against HMGB1 (Abcam, Cambridge, UK), $\beta$-actin (Santa Cruz Biotechnology, Santa Cruz, CA) and horseradish peroxidase-labeled goat anti-rabbit or goat anti-mouse Ig (Jackson Labs,
Bar Harbor, ME) secondary antibodies were used. Enhanced chemiluminescence reagent (Amersham, Buckinghamshire, UK) was used to reveal the signals. Relative band intensities were measured using Image J software (NIH, Bethesda, MD).

Cell viability measurement. As NHNE cells were cultured in airliquid interface system on transwell culture insert, lactate dehydrogenase assay using culture supernatant was applied for viability measurement. For RPMI 2650 cells, conventional CCK8 assay was applied for viability measurement. The viability of NHNE cells was determined based on the amount of released lactate dehydrogenase using CytoTox-ONE ${ }^{\mathrm{TM}}$ Homogeneous Membrane Integrity Assay (Promega, Fitchburg, WI). Identical numbers of NHNE cells were cultured under hypoxic conditions for the indicated durations and $50 \mu \mathrm{l}$ of basal culture medium was transferred to a separate assay plate. Next, $50 \mu \mathrm{l}$ of CytoTox-ONE Reagent was applied to each well. After $10 \mathrm{~min}$, stop solution containing $10 \%$ SDS was added and fluorescence was measured using $560 \mathrm{~nm}$ excitation wavelength and $590 \mathrm{~nm}$ emission wavelength. The viability of RPMI 2650 cells was determined by CCK8 assay (Dojindo Laboratories, Kumamoto, Japan) using culture supernatant following manufacturer's protocol.

DUOX1 and DUOX2 silencing. NHNE cells at passage 1 were incubated with DUOX1 and DUOX2 retrovirus particles. The DUOX1 and DUOX2 inserts were cloned into the LZR retroviral vector and retroviral particles were produced in HEK 293 GpG packaging cells. The LZR retroviral vector and HEK 293 GpG packaging cells were gifted by Dr Ji-Hwan Ryu (Yonsei University, Korea). Culture medium was removed after $24 \mathrm{~h}$ and the NHNE cells were cultured until reaching confluence. Next, the cells were sub-cultured and the NHNE cells (passage 2) were incubated with DUOX1 and DUOX2 retrovirus particles for $24 \mathrm{~h}$. Culture medium was changed daily. Experiments were performed using NHNE cells with air-liquid interface for 14 days. Scrambled short hairpin RNA retrovirus particles were used as a negative control.

Real-time PCR. Total RNA was isolated using TRIzol (Invitrogen, Waltham, MA) and cDNA was synthesized using HighCapacity cDNA Reverse Transcription Kits (Applied Biosystems, Waltham, MA) with random hexamer primers (PerkinElmer Life Sciences, Waltham, MA). Real time-PCR was performed using TaqMan universal PCR master mix (\#4324018, Applied Biosystems). Target-specific probes were constructed. The primers used were as follows: DUOX2 primer: reverse primer: 5' -CGAATCCAGTAGTTGTTGCAGACCCTGAGA-3'; HMGB1: forward primer: $5^{\prime}$-AAGTGAGAGCCAGACGGG-3', reverse primer: $\quad 5^{\prime}$-TCCTTTGCCCATGTTTAATTATTTTC-3'; DUOX1: forward primer: 5'-CGAGAGGACCATGTGTTGGTT-3', reverse primer: $5^{\prime}$-TGCGGGAAAACTTCAGTGG-3'; DUOX2 primer: forward primer: $5^{\prime}$-ATGCTCCGTGCAAGACCAG-3', reverse primer: $5^{\prime}$-. The housekeeping gene $\beta 2$ microglobulin was used as an internal control to normalize HMGB1 expression level, and glyceraldehyde 3 -phosphate dehydrogenase was used as an internal control to normalize DUOX1 and 2 expression level. Data quantification was performed using $\Delta C T$ method and target gene values were expressed as fold increase relative to control.

Immunofluorescence analysis. Cells were fixed with $4 \%$ paraformaldehyde for $20 \mathrm{~min}$ at room temperature. To permeabilize fixed cells, $0.2 \%$ Triton X-100 was applied for $10 \mathrm{~min}$. The samples were blocked in $1 \%$ bovine serum albumin-phosphate-buffered saline for $1 \mathrm{~h}$ at room temperature. Samples were then incubated with a primary antibody against HMGB1 (Abcam) in 1\% bovine serum albumin-phosphate-buffered saline overnight at $4{ }^{\circ} \mathrm{C}$. Alexa Fluor 488- or 594-conjugated secondary antibody (Invitrogen) was added to the cells for $1 \mathrm{~h}$ at room temperature. The cells were mounted with Vecta shield mounting solution (Vector Laboratories, Burlingame, CA). Fluorescence images were acquired using an FV1000 confocal microscope (Olympus, Tokyo, Japan). 
a

Control mucosa

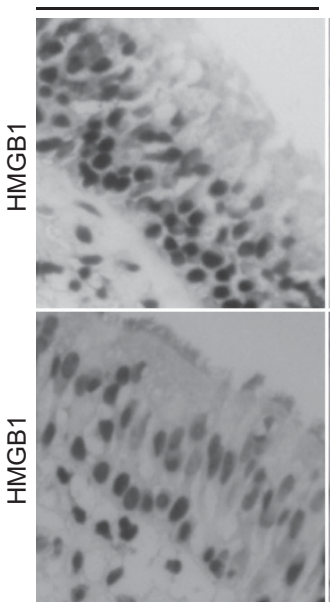

Hypoxic mucosa

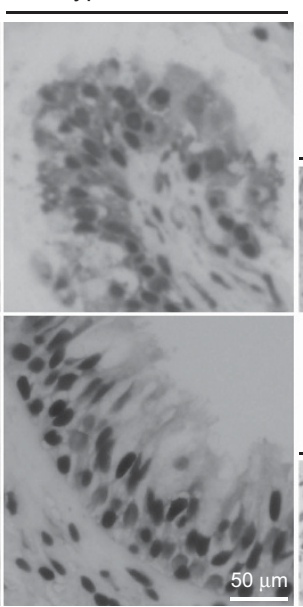

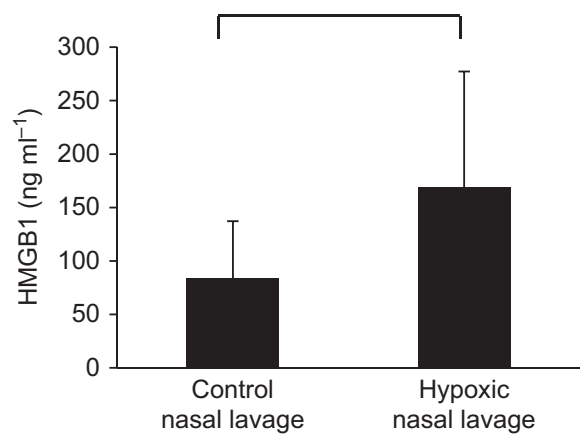

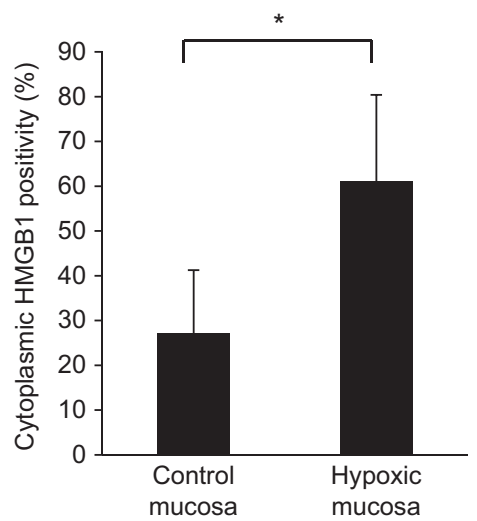

d

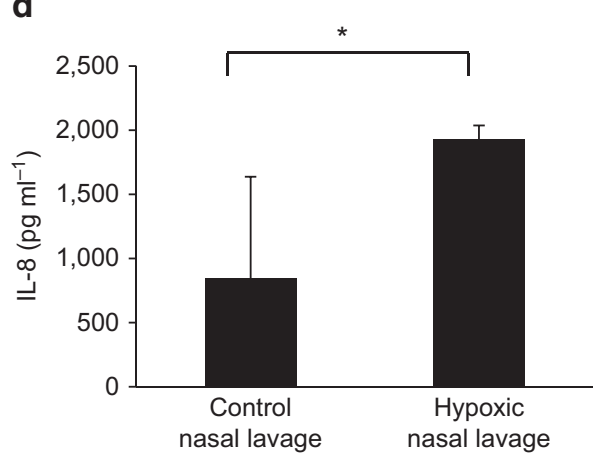

Figure 5 High-mobility group box 1 (HMGB1) is secreted into extracellular space in hypoxic human sinonasal mucosa. (a) Hypoxic sino-nasal mucosa was obtained from patients whose natural ostium was totally obstructed in a preoperative computed tomography (CT) scan and compared with control mucosa from patients whose natural ostium was not obstructed. Immunohistochemistry was performed to compare expression and location of HMGB1 protein in sino-nasal mucosa. (b) Percentage of nasal epithelial cells that show positivity for cytoplasmic HMGB1 were calculated. (c) Nasal lavage fluids were collected from a total of 35 patients. Of these, 18 patients had a completely obstructed maxillary sinus opening and 17 patients had a patent maxillary sinus opening. HMGB1 enzyme-linked immnunosorbent assay (ELISA) was performed to compare the HMGB1 protein secreted into the extracellular space. (d) An ELISA assay for interleukin (IL)-8 was performed to compare the IL-8 in hypoxic and normoxic nasal lavage fluid samples. For all panels, data are shown as mean \pm s.e.m. ${ }^{\star} P<0.05$.

Measurement of intracellular ROS. ROS generated by hypoxia was measured by staining with the fluorescent dye dichlorofluorescin diacetate (Sigma-Aldrich, St Louis, MO). Cells were incubated with Hank's balanced salt solution containing $0.1 \mathrm{~mm}$ dichlorofluorescin diacetate. After $20 \mathrm{~min}$, cells were washed two times with Hank's balanced salt solution and fluorescence was visualized using an FV1000 confocal microscope (Olympus). The effect of an ROS scavenger was evaluated by pretreatment with 100 or $500 \mu \mathrm{M} \mathrm{NAC}$ (Sigma-Aldrich) to culture media $0.5 \mathrm{~h}$ before hypoxic incubation.

Precipitation of secreted HMGB1 protein and treatment of antiHMGB1 antibody. Apical culture medium from control and hypoxiaconditioned NHNE cells was collected and pre-cleared by incubation with protein G-Sepharose beads (Sigma-Aldrich) at $4{ }^{\circ} \mathrm{C}$ for $1 \mathrm{~h}$. The pre-cleared culture medium was then incubated with rabbit antiHMGB1 antibody (Abcam) overnight and anti-HMGB1 antibodybound HMGB1 proteins were removed by precipitation with protein G-Sepharose for $2 \mathrm{~h}$ at $4{ }^{\circ} \mathrm{C}$. This recovered HMGB1 protein was confirmed by western blot analysis. The remaining apical culture supernatants that had been cleared of HMGB1 were collected and reapplied to NHNE cells, to determine the autocrine and paracrine effect of HMGB protein on the secretion of IL- 8 in the NHNE cell culture system.
To inhibit the function of secreted HMGB1 after hypoxia, $20 \mu \mathrm{g} \mathrm{ml}^{-1}$ of anti-HMGB1 neutralizing antibody and isotypematched control antibody (Shino-Test Corp., Tokyo, Japan) was applied to apical culture medium.

Human samples. After approval of the study protocol by the Institutional Review Board of the Yonsei University College of Medicine (4-2014-0139), human sino-nasal mucosa and nasal secretions were obtained from patients who underwent ESS. The presence of hypoxia in each subject was estimated by pre-operative computed tomography scans. By coronal and axial computed tomography images, complete obstruction of ostiomeatal complex unit was supposed to be hypoxic condition and, during operation, we confirmed that ostiomeatal complex unit was obstructed. The mucosa of obstructed sinus was supposed to be hypoxic-conditioned mucosa. ${ }^{25}$ Total 35 nasal secretions and sino-nasal mucosa samples were collected before surgery. All patients gave informed consent before surgery. Patients with a history of atopy/allergic rhinitis and who had taken corticosteroid in the previous 4 weeks were excluded from our study.

Immunohistochemistry. Nasal mucosa samples were obtained using cutting forceps during ESS at the beginning of the procedure. 
Immunohistochemistry was performed using Envision ${ }^{+}$kits (Dako, Carpinteria, CA). Briefly, nasal mucosa blocks were deparaffinized and rehydrated in graded alcohol solutions. After antigen retrieval, blocking was performed with $3 \%$ bovine serum albumin. Primary antibodies were diluted in blocking buffer and incubated overnight at $4{ }^{\circ} \mathrm{C}$, followed by incubation with anti-rabbit immunoglobulin antibody using the Dako Envision ${ }^{+}$kit for 30 min. After washing with TBS, the sections were further incubated at room temperature for $20 \mathrm{~min}$, followed by staining with 3,3'-diaminobenzidine for $10 \mathrm{~min}$. Isotype-matched antibody was used as a control. At least 100 highpower fields were selected randomly and $>100$ cells were counted for each section. Values represent percentages of nasal epithelial cells with HMGB1 cytoplasmic translocation in all nasal epithelial cells counted in each group.

IL-8 measurements. rHMGB1 proteins were produced in mammalian cells (Euk HMGB1; Shino). Flagellin (Invivogen, San Diego, CA) was used as a positive inducer of IL- 8 . The amount of secreted IL- 8 in apical culture supernatant and nasal lavage fluid samples was determined using a commercial ELISA kit (R\&D Systems, Minneapolis, MN). In our IL-8 ELISA assay, minimal detectable dose ranged from 1.5 to $7.5 \mathrm{pg} \mathrm{ml}^{-1}$ and the mean minimal detectable dose was $3.5 \mathrm{pg} \mathrm{ml}^{-1}$.

HMGB1 ELISA of nasal lavage fluids. Nasal lavage fluids were obtained from 35 patients who underwent ESS. Briefly, $5 \mathrm{ml}$ of normal saline was injected into the nasal cavity using a syringe and nasal secretions were suctioned out before intranasal manipulation. We collected nasal lavage fluids in head-down position, because we collected lavage fluid at the beginning of ESS. Therefore, some amount of saline was lost into postnasal airway through nasopharynx. To minimize inter-sample variation, this procedure was performed by one skilled ENT surgeon and we collected regular amount of inserted saline. All secretions were immediately centrifuged to remove blood or mucus and stored at $-70{ }^{\circ} \mathrm{C}$ until use in the experiments. The amount of HMGB1 in these fluids was assessed using the HMGB1 ELISA kit (Shino).

Statistical analysis. Data are presented as mean \pm s.d. In vitro experiments were repeated at least three times and the differences between the two groups were evaluated by independent two-sample $t$-test. The level of HMGB1 and IL-8 between two groups was compared using Mann-Whitney $U$-test. $P$-values $<0.05$ were considered significant. SPSS Statistics 20 software package (IBM, Armonk, NY) was used to perform all statistical analyses.

SUPPLEMENTARY MATERIAL is linked to the online version of the paper at http://www.nature.com/mi

\section{ACKNOWLEDGMENTS}

This research was supported by the National Research Foundation of Korea (NRF) grant funded by the Korean government (MSIP; 2014R1A1A1036052 to HJM) and (MSIP; 2014R1A2A01003385 to $\mathrm{J}-\mathrm{HY}$ ). This research was also supported by Yonsei University FutureLeading Research Initiative of 2014 (2014-22-0131) and the Bio \& Medical Technology Development Program of the NRF, ICT \& Future Planning (2013M3A9D5072551 to C-HK). Clinical implications: These data support that HMGB1 has a role in the pathogenesis of hypoxia-mediated inflammatory diseases in upper airway epithelium through ROS-dependent mechanism.

\section{AUTHOR CONTRIBUTIONS}

HJM planned detailed experiments and C-HK supervised as a correspondence author. Other co-authors participated in maintaining of primary human nasal epithelial cell culture and collecting human samples.

\section{DISCLOSURE}

The authors declared no conflict of interest.

\section{REFERENCES}

1. Lotze, M.T. \& Tracey, K.J. High-mobility group box 1 protein (hmgb1): Nuclear weapon in the immune arsenal. Nat. Rev. Immunol. 5, 331-342 (2005).

2. Bonaldi, T. et al. Monocytic cells hyperacetylate chromatin protein hmgb1 to redirect it towards secretion. EMBO J. 22, 5551-5560 (2003).

3. Hori, O. et al. The receptor for advanced glycation end products (rage) is a cellular binding site for amphoterin. Mediation of neurite outgrowth and coexpression of rage and amphoterin in the developing nervous system J. Biol. Chem. 270, 25752-25761 (1995).

4. Park, J.S. et al. Involvement of toll-like receptors 2 and 4 in cellular activation by high mobility group box 1 protein. J. Biol. Chem. 279, 73707377 (2004).

5. Park, J.S. et al. Activation of gene expression in human neutrophils by high mobility group box 1 protein. Am. J. Physiol. Cell Physiol. 284, C870-C879 (2003).

6. Treutiger, C.J. et al. High mobility group $1 \mathrm{~b}$-box mediates activation of human endothelium. J. Intern. Med. 254, 375-385 (2003).

7. Ladrech, S., Mathieu, M., Puel, J.L. \& Lenoir, M. Supporting cells regulate the remodelling of aminoglycoside-injured organ of corti, through the release of high mobility group box 1. Eur. J. Neurosci. 38, 2962-2972 (2013).

8. Min, H.J. et al. Chaperone-like activity of high-mobility group box 1 protein and its role in reducing the formation of polyglutamine aggregates J. Immunol. 190, 1797-1806 (2013).

9. Tang, D. et al. Endogenous hmgb1 regulates autophagy. J. Biol. Chem. 190, 881-892 (2010).

10. Han, S.J. et al. Hmgb1 in the pathogenesis of ultraviolet-induced ocular surface inflammation. Cell Death Dis. 6, e1863 (2015).

11. Steinke, J.W., Woodard, C.R. \& Borish, L. Role of hypoxia in inflammatory upper airway disease. Curr. Opin. Allergy Clin. Immunol. 8, 16-20 (2008).

12. Wang, G.L., Jiang, B.H., Rue, E.A. \& Semenza, G.L. Hypoxia-inducible factor 1 is a basic-helix-loop-helix-pas heterodimer regulated by cellular 02 tension. Proc. Natl Acad. Sci. USA 92, 5510-5514 (1995).

13. Matsune, S., Kono, M., Sun, D., Ushikai, M. \& Kurono, Y. Hypoxia in paranasal sinuses of patients with chronic sinusitis with or without the complication of nasal allergy. Acta Otolaryngol. 123, 519-523 (2003).

14. Baroody, F.M. Mucociliary transport in chronic rhinosinusitis. Clin. Allergy Immunol. 20, 103-119 (2007).

15. Min, H.J. et al. Level of secreted hmgb1 correlates with severity of inflammation in chronic rhinosinusitis. Laryngoscope 125, E225-E230 (2015).

16. Itoh, T. et al. High-mobility group box 1 expressions in hypoxia-induced damaged mouse islets. Transplant. Proc. 43, 3156-3160 (2011).

17. Hamada, T. et al. Extracellular high mobility group box chromosomal protein 1 is a coupling factor for hypoxia and inflammation in arthritis. Arthritis Rheum. 58, 2675-2685 (2008).

18. Joo, J.H. et al. Dual oxidase 2 is essential for the toll-like receptor 5-mediated inflammatory response in airway mucosa. Antioxid. Redox Signal. 16, 57-70 (2012).

19. Kruger, B. et al. Donor toll-like receptor 4 contributes to ischemia and reperfusion injury following human kidney transplantation. Proc. Nat/ Acad. Sci. USA 106, 3390-3395 (2009).

20. De Perrot, M. et al. Interleukin-8 release during early reperfusion predicts graft function in human lung transplantation. Am. J. Respir. Crit. Care Med. 165, 211-215 (2002).

21. Ye, J. Emerging role of adipose tissue hypoxia in obesity and insulin resistance. Int. J. Obes. (Lond.) 33, 54-66 (2009).

22. Hoppe, G., Talcott, K.E., Bhattacharya, S.K., Crabb, J.W. \& Sears, J.E. Molecular basis for the redox control of nuclear transport of the structural chromatin protein hmgb1. Exp. Cell Res. 312, 3526-3538 (2006).

23. Schiraldi, M. et al. Hmgb1 promotes recruitment of inflammatory cells to damaged tissues by forming a complex with cxcl12 and signaling via cxcr4. J. Exp. Med. 209, 551-563 (2012)

24. Yang, $\mathrm{H}$. et al. Redox modification of cysteine residues regulates the cytokine activity of high mobility group box-1 (hmgb1). Mol. Med 18, 250-259 (2012).

25. Kim, Y.J. et al. Hypoxia-mediated mechanism of muc5ac production in human nasal epithelia and its implication in rhinosinusitis. PLOS ONE 9 , e98136 (2014). 


\section{ARTICLES}

26. Early, S.B., Hise, K., Han, J.K., Borish, L. \& Steinke, J.W. Hypoxia stimulates inflammatory and fibrotic responses from nasal-polyp derived fibroblasts. Laryngoscope 117, 511-515 (2007).

27. Min, H.J., Kim, T.H., Yoon, J.H. \& Kim, C.H. Hypoxia increases epithelial permeability in human nasal epithelia. Yonsei Med. J. 56, 825-831 (2015).

28. Desireddi, J.R., Farrow, K.N., Marks, J.D., Waypa, G.B. \& Schumacker, P.T. Hypoxia increases ros signaling and cytosolic $\mathrm{ca}(2+)$ in pulmonary artery smooth muscle cells of mouse lungs slices. Antioxid. Redox Signal. 12, 595-602 (2010).

29. Schroedl, C., McClintock, D.S., Budinger, G.R. \& Chandel, N.S. Hypoxic but not anoxic stabilization of hif-1alpha requires mitochondrial reactive oxygen species. Am. J. Physiol. Lung Cell Mol. Physiol. 283, L922-L931 (2002).

30. Xie, K. Interleukin-8 and human cancer biology. Cytokine Growth Factor Rev. 12, 375-391 (2001).
31. Shun, C.T., Lin, S.K., Hong, C.Y., Huang, H.M. \& Liu, C.M. Hypoxia induces cysteine-rich 61, vascular endothelial growth factor, and interleukin-8 expressions in human nasal polyp fibroblasts: an implication of neutrophils in the pathogenesis of nasal polyposis. Am. J. Rhinol. Allergy 25, 15-18 (2011).

32. Bianchi, M.E. Hmgb1 loves company. J. Leukoc. Biol. 86, 573-576 (2009).

33. Shahriary, C.M., Chin, T.W. \& Nussbaum, E. Respiratory epithelial cell lines exposed to anoxia produced inflammatory mediator. Anat. Cell Biol. 45, 221-228 (2012).

34. Kim, C.H. et al. Muc8 as a ciliated cell marker in human nasal epithelium. Acta Otolaryngol. 125, 76-81 (2005).

35. Lee, S.N., Lee, D.H., Lee, M.G. \& Yoon, J.H. Proprotein convertase $5 / 6 a$ is associated with bone morphogenetic protein-2-induced squamous cell differentiation. Am. J. Respir. Cell Mol. Biol. 52, 749-761 (2015). 